Effects of radi o-frequency dri vi ng power, gas pressure, and nitrogen seeding on the transi ti on dynamics i $n$ argon i nduct i vel y coupl ed pl asmas

\begin{tabular}{|l|l|}
\hline 著者 & $\begin{array}{l}\text { Razzaka M Abdur, Takamur a Shui chi, Uesugi } \\
\text { Yoshi hi ko }\end{array}$ \\
\hline $\begin{array}{l}\text { j our nal or } \\
\text { publ i cat i on titl e }\end{array}$ & Journal of Appl i ed Physi cs \\
\hline vol une & 96 \\
\hline number & 9 \\
\hline page range & $4771-4776$ \\
\hline year & 2004 11- 01 \\
\hline URL & ht t p: //hdl . handl e. net /2297/14407 \\
\hline
\end{tabular}




\title{
Effects of radio-frequency driving power, gas pressure, and nitrogen seeding on the transition dynamics in argon inductively coupled plasmas
}

\author{
M. Abdur Razzak ${ }^{\text {a) }}$ and Shuichi Takamura \\ Department of Energy Engineering and Science, Graduate School of Engineering, Nagoya University, \\ Furo-cho, Chikusa-ku, Nagoya 464-8603, Japan \\ Yoshihiko Uesugi \\ Department of Electrical and Electronic Engineering, Kanazawa University 2-40-20 Kodatsuno, Ishikawa \\ 920-8667, Japan
}

(Received 26 January 2004; accepted 16 July 2004)

\begin{abstract}
The influences of $\mathrm{rf}$ driving power, neutral gas pressure, and nitrogen seeding on the electrostatic-to-electromagnetic $(E-H)$ mode transition dynamics in radio-frequency argon inductively coupled plasmas (ICPs) in a pressure range of $50-100 \mathrm{kPa}$ are investigated, both experimentally and theoretically. The $E-H$ mode transition dynamics and its characteristic transition time scale are investigated by observing the high-speed imaging (13 $500 \mathrm{fps}$ ) as well as the temporal change of plasma loading impedance. The experimental results reveal that the $E-H$ mode transition time is not fixed at any operating conditions rather it depends on some important parameters such as the rf driving power, neutral gas pressure, gas type. It is found that the $E-H$ mode transition time depends on the unique parameter $E_{\theta} / p$; the so-called effective induced electric field, rather than the independent parameter: the rf power or neutral gas pressure. It is also found that longer $E-H$ mode transition time is required to ignite the high-pressure Ar- $\mathrm{N}_{2}$ plasmas with a $2.5 \%-10 \% \mathrm{~N}_{2}$ seeding than that of pure Ar plasmas with the same operating conditions. The experimental results are compared with that of the recently developed theoretical models, and a good agreement is found between them. () 2004 American Institute of Physics. [DOI: 10.1063/1.1790066]
\end{abstract}

\section{INTRODUCTION}

It has already been established that the typical radio frequency (rf) inductively coupled plasmas (ICPs) are operated in two well-known modes: A capacitively coupled or electrostatic mode ( $E$ mode) and inductively coupled or electromagnetic mode ( $H$ mode). Recently, attention has been renewed to investigate the dynamics of this $E-H$ discharge mode transition and many theoretical and experimental works have been conducted recently and in the past decade, but mainly for low-pressure (milliTorr range) discharges,${ }^{1-6}$ because of simple and easy generation, and diagnostics of the lowpressure ICPs. But, when operated at high pressures (around $1 \mathrm{~atm}$ or more), initial startup is one of the most important issues to ignite the discharge of induction plasmas, as the starting of high-pressure $\mathrm{rf}$ induction plasma torches are hard, and a high-voltage initiation is usually required. ${ }^{7}$ So far several techniques such as, $\mathrm{dc}$ arc jet ${ }^{8}$ and low-pressure small-size plasma torch, ${ }^{9}$ are used to initiate the highpressure rf inductive discharges. But, these hybrid plasma torches characteristically operate at power levels of about $50-100 \mathrm{~kW}$ or even more, where the dimensions of the second-stage, high-power unit make it difficult to initiate and operate in a stable manner. ${ }^{7}$ Therefore, the initiation of highpressure rf inductive discharges with moderate rf power of a few kilowatts is an interesting and challenging issue, and with that in mind, we have investigated the high-pressure (up to $1 \mathrm{~atm})$ ICP discharges at the ignition stage.

\footnotetext{
${ }^{a)}$ Electronic mail: razzak@ees.nagoya-u.ac.jp
}

The hysteresis and $E-H$ discharge mode transition in low pressure ( $\sim 150$ mTorr) ICPs have been reported by Cunge et al. ${ }^{1}$ and Turner and Lieberman, ${ }^{2}$ taking the power balance argument and possible nonlinear mechanisms into account for sustaining the plasmas. Three-dimensional optical emission spectroscopy with computer tomography is used by Myoshi, Petrovic, and Makabe ${ }^{3}$ to explain the $E-H$ mode transition in low-pressure ( $\sim 300$ mTorr) ICPs. The dynamics of mode transition in low-pressure $(50 \mathrm{mTorr})$ low frequency $(460 \mathrm{kHz}) \mathrm{Ar}$ and $\mathrm{N}_{2}$ ICPs are reported by Ostrikov et $a l .{ }^{4}$ by observing the plasma images using a charge coupled device (CCD) camera with a long exposure time of $\approx 17 \mathrm{~ms}$. The initial startup of high-pressure Ar inductive discharges was reported by Uesugi et al. ${ }^{10}$ by observing the plasmas using only the CCD camera, and also without explaining the physical mechanism and dynamics of $E-H$ mode transition. However, by using the CCD camera, it is very difficult to observe the $E-H$ discharge mode transition mechanism since the transition time is very short. In the above mentioned papers, ${ }^{1-10}$ the $E-H$ discharge mode transition time, which is very important factor to be taken into account for analyzing the fundamental physics of highpressure ICP generation, is not considered or analyzed. In our previous work, ${ }^{11}$ the $E-H$ mode transition dynamics of Ar ICP in atmospheric pressure are observed by high-speed and CCD camera imaging, and the mode transition times are estimated by three different proposed models. However, the rf driving power, neutral gas pressure, and the $\mathrm{N}_{2}$ seeding has remarkable influence on the generation of high-pressure induction plasmas. ${ }^{4,6,712}$ Therefore, the motivation of this 


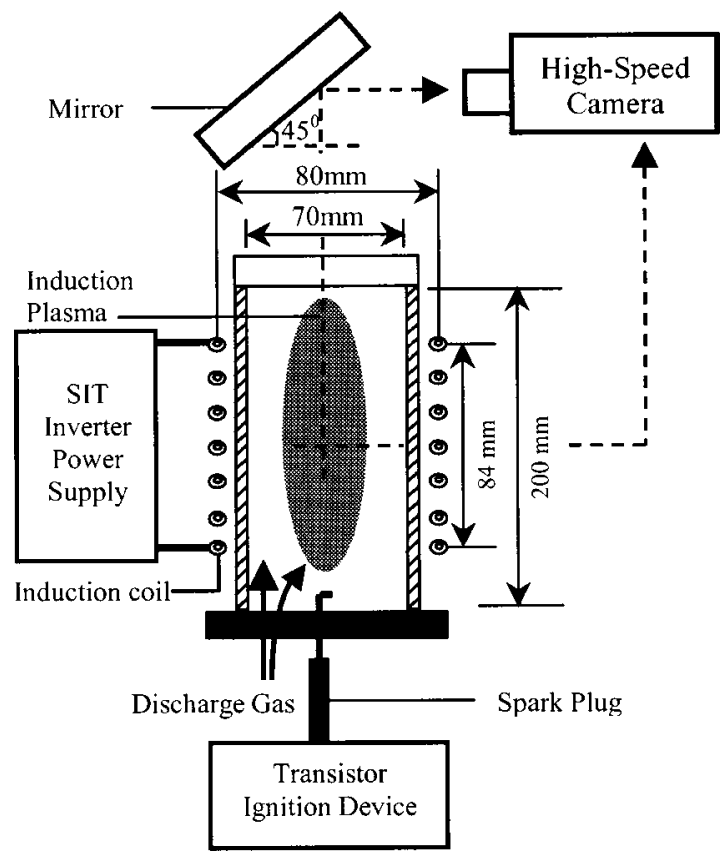

FIG. 1. Schematic diagram of experimental setup.

work is to investigate the influences of $\mathrm{rf}$ driving power, neutral gas pressure, and $\mathrm{N}_{2}$ seeding $(2.5 \%-10 \%)$ on the $E-H$ mode transition dynamics as well as on the mode transition time in high-pressure (up to $1 \mathrm{~atm}$ ) Ar induction plasmas in a frequency range of $1-1.2 \mathrm{MHz}$ and moderate rf power of about $1-5 \mathrm{~kW}$. The experimental results are compared with that of the recently developed theoretical model calculations. ${ }^{11}$

\section{EXPERIMENTS}

The schematic diagram of experimental setup is depicted in Fig. 1. The inverter dc input voltage and current, rf output voltage and current, and rf coil current are measured to analyze the $E-H$ mode transition time. The plasmas are sustained in a cylindrical Pyrex glass chamber with an internal diameter of $70 \mathrm{~mm}$ and length of $200 \mathrm{~mm}$ by applying a static induction transistor (SIT) inverter power source, the equivalent circuit of which along with rf induction coil is shown in Fig. 2. The rating of frequency range and maximum output power of the SIT inverter is $0.2-1.7 \mathrm{MHz}$ and $20 \mathrm{~kW}$, respectively. An induction coil consisting of seven turns of a copper tube of $1 / 4$ inch outer diameter is used as the loop antenna. Ar gas is injected both axially and spirally into the torch vessel with a total flow rate of $20 \mathrm{lpm}$. The neutral pressure measured with a total pressure gauge is varied from

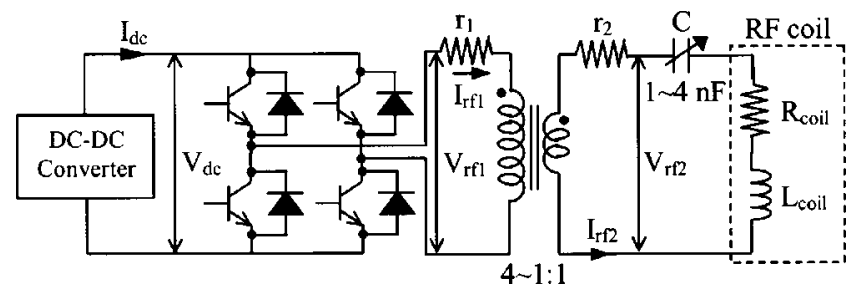

FIG. 2. Equivalent circuit diagram of SIT inverter power source and $\mathrm{rf}$ induction coil (one of the six units of SIT inverters is shown in the figure).
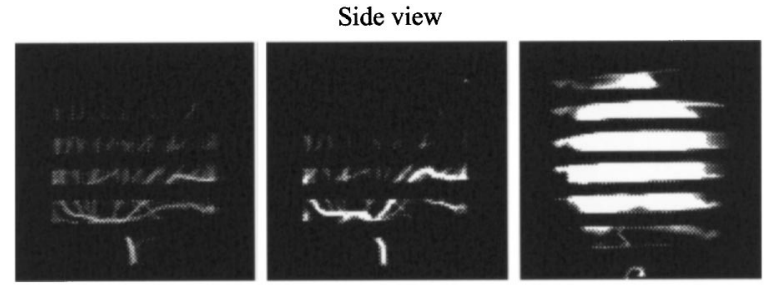

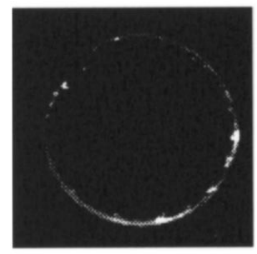

(a)

$0.4 \mathrm{~ms}$

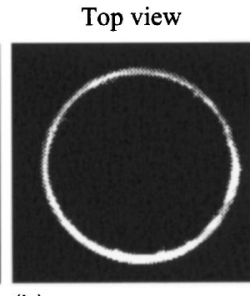

(b)

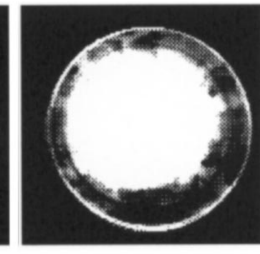

(c)

$8.0 \mathrm{~ms}$
FIG. 3. Observation of $E-H$ mode transition dynamics by high-speed imaging in atmospheric pressure.

$50-100 \mathrm{kPa}$ by using a mechanical rotary pump. The rf power level, which is limited by the cooling capability of the system, is modulated with a $100-5000 \mathrm{~ms}$ square wave pulse without applying any cooling system. Repetitive spark discharge, using the spark discharge technique, ${ }^{10,12}$ with a repetition frequency of $500 \mathrm{~Hz}$ and duration of $20-30 \mathrm{~ms}$, is applied simultaneously with the rf pulse to initiate the discharge. This task is performed by using a typical automobile spark plug, placed at the center of the bottom flange of the discharge chamber with a high-voltage transformer circuit as shown in Fig. 1. An L-C matching network is employed to optimize the power coupling efficiency. A "FASTCAMultima SE" high-speed camera with a frame speed of $13500 \mathrm{fps}$ is used to observe the mode transition dynamics. The side view images are taken by setting the camera perpendicular to the axis of the discharge chamber, while the top views are taken by setting a mirror on the top of the discharge chamber and $45^{\circ}$ inclined to that of the discharge axis as shown in Fig. 1.

\section{E-H MODE TRANSITION DYNAMICS: EXPERIMENTAL OBSERVATIONS}

The $E-H$ discharge mode transition dynamics together with the transition mechanisms and transition time are investigated by observing the high-speed imaging as well as the temporal plasma loading resistance and reactance. They are described below.

\section{A. High-speed Imaging}

In our previous work, ${ }^{11}$ the side view images were taken by high-speed camera with a frame speed of $4500 \mathrm{fps}$ and bottom view images by conventional $\mathrm{CCD}$ camera with which, however, it is difficult to estimate the $E$ - $H$ mode transition time with high accuracy. Therefore, here we observe both the side and the top view images by only the high-speed imaging with a higher frame speed of $13500 \mathrm{fps}$. The typical examples of images are shown in Fig. 3, where the formation of $E$ discharge, $E-H$ mode transition, and the steady state plasmas are clearly observed. The physical mechanisms of 
the $E-H$ mode transition dynamics can briefly be described as follows (for more details with mathematical analysis, the readers are referred to Ref. 11): A strong axial electrostatic field $E_{z}$, the average intensity of which is about $100 \mathrm{kV} / \mathrm{m}$ in atmospheric pressure, develops at the initial phase of $\mathrm{rf}$ breakdown due to high rf voltage on the induction coil. Highly mobile electrons are accelerated and collide with the neutral particles, and picked up energy from this applied electrostatic field to excite and ionize the working gas atoms (we assume that the electron-impact ionization is the dominant process in the present condition). However, the electron energy gain by the single electron-neutral collision is not high enough to ionize the working gas. At this stage, collisional heating occurs due to the strong axial electrostatic field, which gives enough energy to the electrons for ionization to produce electron-ion pairs. The newly formed electrons together with the primary ones repeat this process and produce more free electrons. Therefore, cumulative ionizations takes place along the direction of the electrostatic field thereby developing the multiple streamerlike discharge ( $E$ discharge) paths at the bottom and very close to near the surface of the discharge chamber [Fig. 3(a)] due to the stronger $E_{z}$ near the torch surface.

Then, the induced electric field $E_{\theta}$, which is found about $2.5 \mathrm{kV} / \mathrm{m}$ in atmospheric pressure, helps to connect the discharge paths among the streamers. After the ionization, the newly formed electrons move diffusively in the azimuthal direction, because the phase of these electrons may be the same as or opposite to that of the old ones since the phase is a random number between 0 and $2 \pi$. These newly formed electrons together with the primary ones repeat the ionization process and produce more free electrons. This process continues until an electrically conducting bridge between neighboring streamers has been formed.

The motion of electrons, on the other hand, is very much faster compared to that of the ions. Therefore, alternatively to the previous diffusive model, the effective motion can be considered as the ambipolar drift motion by assuming that such an ionized plasma is convected with the ion mass and that of the effective electron kinetic energy, which will be described later in Sec. IV. The induced electric field, therefore, promotes diffusive and/or convective drift motion for electrons in the azimuthal direction. These energetic electrons produce ionization and make electrically conducting bridges between the neighboring streamers by the diffusive and/or convective process described above, and transform the streamers into the ring-shaped azimuthal discharge $(H$ discharge) paths [Fig. 3(b)].

In the primary stage of $H$ discharge still some streamers exist even after transforming it into the ring shaped plasma as shown in the side view of Fig. 3(b). But these streamers disappear as soon as the azimuthal current is induced by the electrically conductive ring, thereby injecting the Joule power into the ring-shaped plasma. Therefore, for the time being, $H$ discharge develops upward thereby forming the steady state plasmas [Fig. 3(c)] due to this Joule heating with the azimuthal rf current. From Fig. 3, it is seen that the

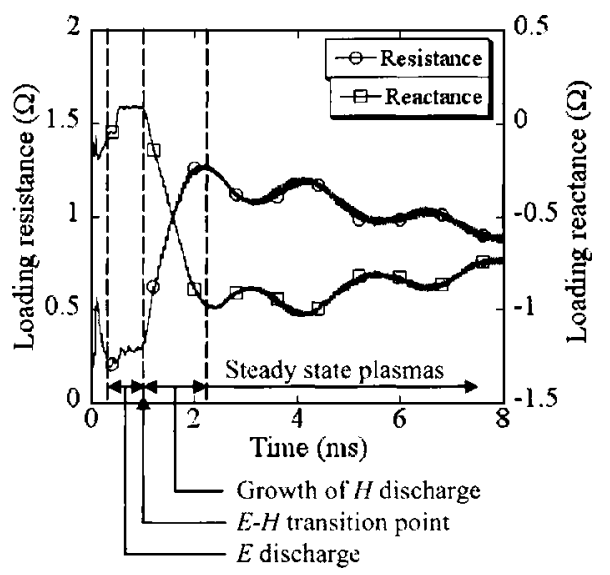

FIG. 4. Observation of $E-H$ mode transition by analyzing the plasma loading resistance and reactance in atmospheric pressure.

discharges develop near the inner surface of the discharge vessel because of the stronger $E_{z}$ and $E_{\theta}$ near the torch surface than the center of the chamber.

\section{B. Plasma Loading Impedance}

In our previous work, ${ }^{11}$ we measured the dc plasma loading impedance obtained by the input dc voltage and current of the SIT inverter circuit. But for better understanding, it is customary to observe the complex rf loading impedance (both resistance and reactance) since the rf plasma loading resistance is the crucial quantity for heating mechanism and power coupling in induction plasma. ${ }^{6}$ Therefore, here we measure the temporal plasma loading resistance and reactance at the ignition stage, obtained by the ratio of the output rf voltage to the current of SIT inverter circuit, to investigate the $E-H$ mode transition dynamics. The complex form of loading impedance looking from the primary circuit (Fig. 2) can be written as

$$
Z_{1}=R+j X=\frac{X_{\mathrm{rf} 1}}{\mathrm{I}_{\mathrm{rf} 1}}
$$

where $V_{\text {rf1 }}$ and $I_{\text {rf1 }}$ are the primary circuit rf voltage and current, respectively, $R$ is the total circuit resistance, and $X$ is the total circuit reactance. Therefore, the plasma loading impedance can be calculated by the transformer turn ratio (assume an ideal one) as

$$
Z_{2}=\left(\frac{n_{2}}{n_{1}}\right)^{2} \frac{V_{\mathrm{rf} 1}}{I_{\mathrm{rf} 1}}
$$

where $n_{1}$ and $n_{2}$ are the numbers of turns of the transformer in the primary and secondary side, respectively.

Figure 4 shows the temporal rf plasma loading resistance and reactance, calculated by the inverter output voltage and current using Eq. (2). The timing of the respective resistance and reactance of the discharge development of Ar plasmas shown in Fig. 3 are indicated in Fig. 4. It is noticed that the plasma loading resistance at the start of ignition, i.e., in the $E$ discharge region remains almost constant and is not high enough for plasma heating. But, the loading resistance increases remarkably after the $E$ - $H$ mode transition, i.e., in the region of the development of $H$ discharge since the rf coil 
current decreases in this region and the steady state plasmas develop as shown in Fig. 4. On the other hand, the loading reactance of the generated plasmas is slightly positive in the $E$ mode region. Because, we adjust the matching network to have the system a slightly inductive L-C resonant condition in order to suppress the switching losses of the inverter elements. However, the reactance decreases after the $E-H$ mode transition, since the streamers disappear in this region, an electrically conducting ring forms and consequently, an induction current flows in the azimuthal direction, but opposite to that of the rf coil current. As a result, the magnetic flux decreases by this plasma reaction, meaning a reduction of coil inductance and hence the reactance.

The development of $E$ discharge, $E-H$ mode transition, development of $H$ discharge, and finally the formation of steady state thermal plasmas can be seen clearly from the dynamic plasma loading resistance and reactance since the slope of the curve changes at the start of every mode as indicated in Fig. 4. From the experimental observations by fast camera imaging and plasma loading impedance, the $E-H$ discharge mode transition times, which correspond to the duration of $E$ discharge, are found to be about $200-800 \mu \mathrm{s}$ at a rf power of about $3.5 \mathrm{~kW}$ and pressure range of $50-100 \mathrm{kPa}$.

\section{EFFECTS OF rf POWER NEUTRAL GAS PRESSURE, AND NITROGEN SEEDING ON THE $E-H$ MODE TRANSITION DYNAMICS}

In our previous work, ${ }^{11}$ we proposed three different plasma models to estimate the $E-H$ mode transition time. The first two of them were based on the diffusion model and the last one was based on the drift model. According to these models, Eqs. (22), (25), and (29) of Ref. 11, to estimate the $E-H$ mode transition times, with a unit in second can be rewritten as

$$
\begin{aligned}
& \tau_{c 1}=p d_{\theta}^{2}\left(1.5 \times 10^{-3}\right), \\
& \tau_{c 2}=\frac{d_{\theta}^{2}}{\left(\mid E_{\theta} / p\right)^{2}}\left(45 \times 10^{-3}\right), \\
& \tau_{c 3}=\frac{d_{\theta}}{\left(\left|E_{\theta}\right| / p\right)}\left(5.5 \times 10^{-3}\right),
\end{aligned}
$$

where $d_{\theta}$ is the distance between neighboring streamers in the azimuthal direction in meter, which is found to be about $2.5 \times 10^{-3} \mathrm{~m}$ in an atmospheric pressure, $p$ is the neutral gas pressure in pascal, and $\left|E_{\theta}\right|$ is the amplitude of the induced electric field in $\mathrm{V} / \mathrm{m}$, which is given by ${ }^{11}$

$$
\left|E_{\theta}\right|=\frac{\mu_{0} \omega r n_{a}\left|I_{\mathrm{rf}}\right|}{2},
$$

where $\omega$ is the driving angular frequency, $r$ is the radius of torch of inner surface, $n_{a}$ is the number of antenna turns per meter, and $\left|I_{\mathrm{rf}}\right|$ is the amplitude of the rf coil current.

From Eqs. (3)-(5), it is seen that the $E-H$ mode transition time is directly proportional to the neutral gas pressure or its square, while inversely proportional to the induced electric field or its square assuming the interstreamer dis-
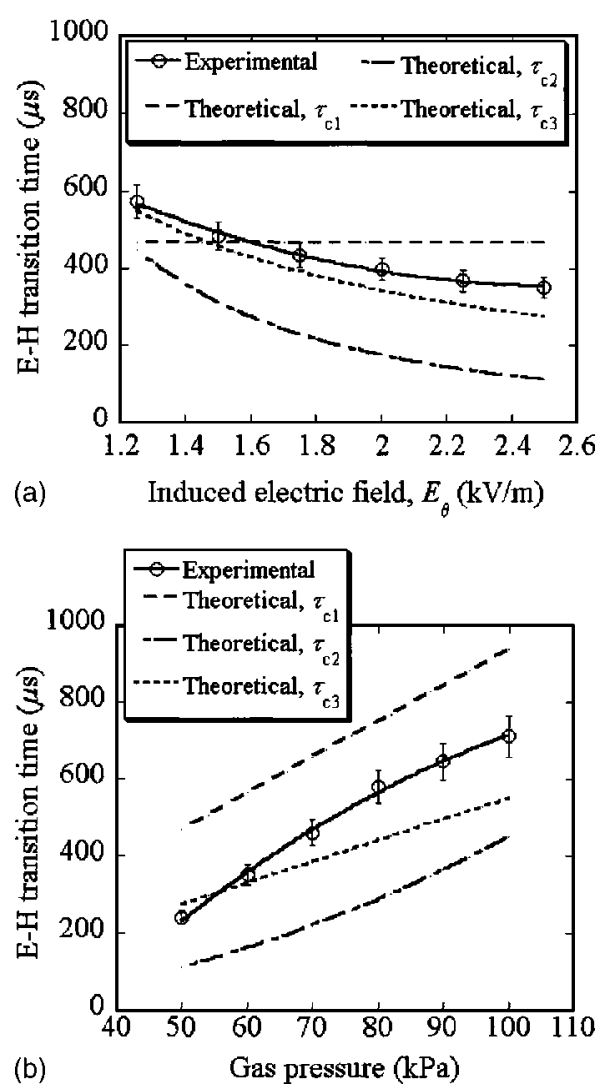

FIG. 5. Effects of azimuthal electric field (a), and neutral gas pressure (b) on the $E-H$ mode transition time. Comparison between experimental results and that of the theoretical model calculations.

tance a constant. Since the applied rf power (or rf coil current) determines the induced azimuthal electric field [Eq. (6)], we will here consider the induced azimuthal electric field, $E_{\theta}$ to investigate the effects of rf driving power on the $E-H$ mode transition time. We also consider the rf coil current by neglecting the plasma reaction, which reduces the coil inductance by the induced azimuthal current, since we are considering the coil current during the mode transition time period before the growth of $H$ discharge.

The $E-H$ mode transition time as a function of the induced electric field at a fixed gas pressure of $50 \mathrm{kPa}$ is shown in Fig. 5(a), which indicates that the mode transition time decreases by increasing the induced electric field (or rf power). This is because the increment of the rf power increases both the axial as well as the azimuthal electric fields [Eq. (6)] which help an easy and fast ionization and accelerate the $E-H$ mode transition. Besides, with an increase of the rf power, the effective electron kinetic energy $\left(W_{\theta}\right.$ $\left.=m_{e} v_{\theta}^{2} / 2\right)$ and thus the ion sound velocity $\left(c_{s}=\sqrt{W_{\theta} / m_{i}}\right)$ increases, and consequently, the transition time decreases by assuming the interstreamer distance a constant. The $E-H$ mode transition time, therefore, decreases with an increase of the rf power with a fixed operating gas pressure.

The neutral gas pressure dependence of the mode transition time, on the other hand, is shown in Fig. 5(b), from which it is noticed that the $E-H$ mode transition time increases by increasing the operating gas pressure at a fixed $\mathrm{rf}$ power of about $3.5 \mathrm{~kW}$. This is because, by increasing the gas pressure, the electron-neutral collision frequency in- 


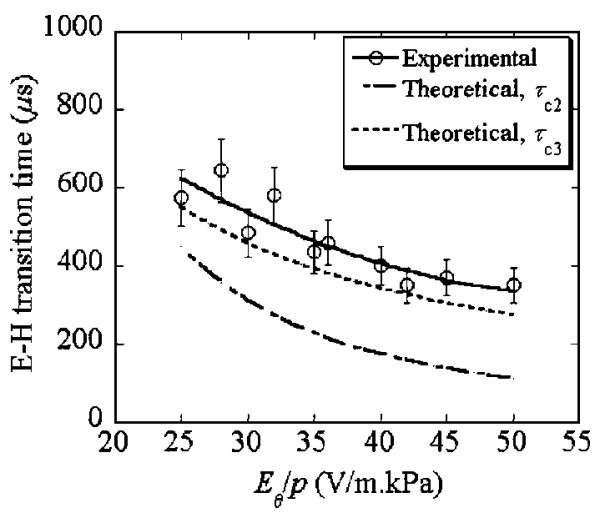

FIG. 6. Effective induced electric field dependence of the $E-H$ mode transition time. Comparison between experimental results and that of the theoretical model calculations.

creases, which decreases the electron oscillating velocity. A decrease of the electron oscillating velocity means a decrease of the effective electron kinetic energy and thus the ion sound velocity, and consequently, an increase of the transition time. The longer time, therefore, is needed for the $E-H$ mode transition with an increase of the operating gas pressure.

From Fig. 5, it is seen that the experimental results agree reasonably with that of the theoretical model calculations using Eqs. (3)-(5). The discrepancy appears because the diffusive and/or convective losses, which increase with an increase of the rf power and neutral gas pressure, are not included in the theoretical model.

From Eqs. (4) and (5), it is seen that the $E-H$ mode transition time is inversely proportional to the so-called effective induced rf electric field, $E_{\theta} / p$ or its square. Therefore, it is customary to consider the combined effect of both the $\mathrm{rf}$ power and the neutral gas pressure on the $E-H$ mode transition time. The $E-H$ mode transition time as a function of the effective induced azimuthal electric field $E_{\theta} / p$ is depicted in Fig. 6, from where it is seen that the mode transition time decreases with an increase of the effective induced electric field. However, from the experimental results, it is not clear whether the transition time is inversely proportional to the parameter $E_{\theta} / p$ or its square. But, the tendency of the experimental results and that of the theoretical model calculations are the same, i.e., transition time decreases with an increase of $E_{\theta} / p$, and a reasonable agreement found between the theoretical and that of the experimental results. It is, therefore, remarked that the $E-H$ mode transition time depends on the unique parameter $E_{\theta} / p$ rather than the independent parameter of the rf power or the neutral gas pressure. However, the estimated results using the drift model [Eq. (5)] are in good agreement with that of the experimental results than other two models (Figs. 5 and 6). Therefore, the drift model is considered to be the most convincing one among them.

Finally, the plasma loading resistance of $\mathrm{Ar}$ and $\mathrm{Ar}-\mathrm{N}_{2}$ plasma with a $5 \% \mathrm{~N}_{2}$ seeding in a neutral gas pressure of $80 \mathrm{kPa}$ and rf power of about $5 \mathrm{~kW}$ is shown in Fig. 7. By observing the plasma loading resistances, the $E-H$ mode transition time for Ar plasma is found to be about $600 \mu \mathrm{s}$,

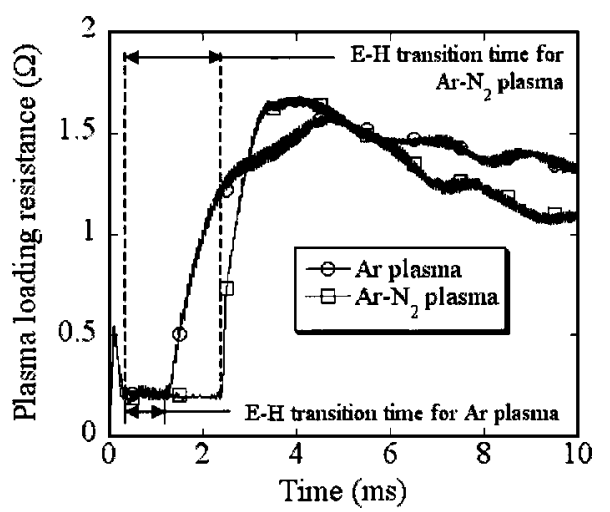

FIG. 7. Effect of $\mathrm{N}_{2}$ seeding on the $E-H$ mode transition time by observing the rf plasma loading resistance.

while about $1700 \mu$ s for Ar- $\mathrm{N}_{2}$ plasma with the same operating conditions. The percentage of $\mathrm{N}_{2}$ seeding, therefore, clearly affects on the initiation time and consequently on the $E-H$ mode transition. With higher $\mathrm{N}_{2}$ seeding, the $E-H$ mode transition time becomes higher and vice versa with the other parameters remaining unchanged. This is because, plasma losses become higher with increasing the percentage of $\mathrm{N}_{2}$ seeding on pure Ar plasma due to high-power consumption for the formation of molecular ions $\mathrm{N}_{2}{ }^{+}$and dissociation of $\mathrm{N}_{2}$ molecules. ${ }^{4}$

\section{CONCLUSIONS}

In this paper, the influences of rf power, neutral gas pressure, and nitrogen seeding on the $E-H$ mode transition time together with the transition dynamics in rf argon inductively coupled plasmas up to atmospheric pressure are investigated, both experimentally and theoretically. The experimental results reveal that the $E-H$ mode transition time is not fixed at all operating conditions rather it depends on some important parameters such as the rf driving power (or induced electric field), neutral gas pressure, gas types etc. The effects of $\mathrm{rf}$ power and neutral gas pressure on the mode transition time are investigated separately, but it is found that the $E-H$ mode transition time depends on the unique parameter $E_{\theta} / p$ rather than on the independent parameters, rf power or neutral gas pressure (Fig. 6). A reasonable agreement is found between the theoretical model calculations and experimental results. However, the estimated results using the drift model [Eq. (5)] are in better agreement with that of the experimental ones than other two models (Figs. 5 and 6). From the experimental results it is also found that a longer $E-H$ mode transition time is required to ignite the high-pressure $\operatorname{Ar}-\mathrm{N}_{2}$ plasmas with a $2.5 \%-10 \% \mathrm{~N}_{2}$ seeding, than that of pure Ar plasmas with the same operating conditions.

\section{ACKNOWLEDGMENTS}

The authors would like to express their sincere thanks to the program committee of NIFS (National Institute for Fusion Science), Japan for their cooperation in providing the FASTCAM-ultima SE high-speed camera during the experiments. They also thank Dr. Ohno for his fruitful advice dur- 
ing discussion and Takagi for his technical assistance during experimental setup.

${ }^{1}$ G. Cunge, B. Crowley, D. Vender, and M. M. Turner, Plasma Sources Sci. Technol. 8, 576 (1999).

${ }^{2}$ M. M. Turner and M. A. Lieberman, Plasma Sources Sci. Technol. 8, 313 (1999).

${ }^{3}$ Y. Myoshi, Z. Lj. Petrovic, and T. Makabe, IEEE Trans. Plasma Sci. 30, 130 (2002).

${ }^{4}$ K. Ostrikov, E. Tsakadze, J. Ning, L. Jidong, R. Storer, and S. Xu, IEEE Trans. Plasma Sci. 30, 128 (2002).

${ }^{5}$ U. Kortshagen, N. D. Gibson, and J. E. Lawler, J. Phys. D 29, 1224 (1996).

${ }^{6}$ N. S. Yoon, B. C. Kim, J. G. Yang, and S. M. Hwang, IEEE Trans. Plasma
Sci. 26, 190 (1998).

${ }^{7}$ J. Reece Roth, Industrial Plasma Engineering-Volume 1: Principles (Institute of Physics Publishing, Bristol, 1995).

${ }^{8}$ T. Uesugi, O. Nakamura, T. Yoshida, and K. Akashi, J. Appl. Phys. 64, 3874 (1988).

${ }^{9}$ T. Yoshida, T. Tani, H. Nishimura, and K. Akashi, J. Appl. Phys. 54, 640 (1983).

${ }^{10}$ Y. Uesugi, T. Adachi, K. Kondo, and S. Takamura, Trans. IEE Japan. 122A, 461 (2002).

${ }^{11}$ M. A. Razzak, K. Kondo, Y. Uesugi, N. Ohno, and S. Takamura, J. Appl. Phys. 95, 427 (2004).

${ }^{12}$ M. A. Razzak, K. Kondo, Y. Uesugi, and S. Takamura, Proceedings of the Annual Meeting of the Fundamentals and Materials Society of IEE Japan, 2002 (IEEJ, Tokyo, 2002), p. 112. 